\title{
Urinary Nerve Growth Factor as a Potential Biomarker of Treatment Outcomes in Overactive Bladder Patients
}

\author{
Yoon Seok Suh ${ }^{1}$, Kwang Jin $\mathrm{Ko}^{2}$, Tae Heon Kim ${ }^{3}$, Hyo Serk Lee ${ }^{4}$, Hyun Hwan Sung ${ }^{2}$, Won Jin Cho ${ }^{5}$ Kyu-Sung Lee ${ }^{2,6}$ \\ ${ }^{1}$ Department of Urology, Center for Prostate Cancer, Research Institute and Hospital of National Cancer Center, Goyang, Korea \\ ${ }^{2}$ Department of Urology, Samsung Medical Center, Sungkyunkwan University School of Medicine, Seoul, Korea \\ ${ }^{3}$ Department of Urology, Samsung Changwon Hospital, Sungkyunkwan University School of Medicine, Changwon, Korea \\ ${ }^{4}$ Department of Urology, Cheil General Hospital and Women's Healthcare Center, Dankook University College of Medicine, Seoul, Korea \\ ${ }^{5}$ Department of Urology, Chosun University Hospital, Chosun University School of Medicine, Gwangju, Korea \\ ${ }^{6}$ Department of Medical Device Management and Research, SAIHST, Sungkyunkwan University, Seoul, Korea
}

Purpose: The aim of this study was to investigate urinary nerve growth factor (NGF) as a biomarker of treatment efficacy and recurrence in overactive bladder $(\mathrm{OAB})$ patients.

Methods: We enrolled $189 \mathrm{OAB}$ subjects who visited our outpatient clinic from February 2010 to February 2015. All subjects with $\mathrm{OAB}$ received antimuscarinic treatment. A 3-day voiding diary and questionnaire were collected from each patient. Urinary levels of NGF were evaluated at baseline, the beginning of antimuscarinic treatment, and the end of antimuscarinic treatment. Urinary NGF was normalized to urine creatinine (Cr). Between-group comparisons of baseline characteristics were made using the Mann-Whitney U-test. Multivariate logistic regression analyses were used to predict responses to anticholinergic treatment and recurrence. The Wilcoxon signed-rank test with the Bonferroni correction was used for intragroup comparisons. A receiver operating characteristic curve was used to analyze the utility of this biomarker.

Results: Urinary levels of NGF/Cr tended to decrease in patients who responded to treatment $(n=62)$, but this was not significant $(\mathrm{P}=0.260)$. Urinary NGF levels were higher at baseline in patients who did not experience recurrence than in those who $\operatorname{did}(P=0.047)$. In those who did not experience recurrence $(n=29)$, urinary NGF/Cr decreased at the end of treatment compared to baseline, and this reduction was maintained at 12 weeks after the end of treatment $(\mathrm{P}<0.05)$.

Conclusions: Urinary NGF is a potential biomarker for predicting the outcome of antimuscarinic treatment in OAB patients. This may provide useful information when deciding to stop antimuscarinic treatment in responders.

Keywords: Urinary bladder, Overactive; Biomarkers; Nerve growth factor

- Grant/Fund support: The research was supported by a Samsung Biomedical Research Institute grant, \#SBRI C-B0-227, and a grant of the Korea Health Technology R\&D Project through the Korea Health Industry Development Institute (KHIDI), funded by the Ministry of Health \& Welfare, Republic of Korea (grant number: HI14C3229).

- Research Ethics: This study was approved by the Institutional Review Board of Samsung Medical Center (approval number: 2010-05-106) and was registered at http://clinicaltrials.gov (ClinicalTrials.gov ID: NCT01499069). Written informed consent was obtained from all subjects.

- Conflict of Interest: No potential conflict of interest relevant to this article was reported.

\section{- HIGHLIGHTS}

- Urinary NGF levels were higher at baseline in patients who did not experience recurrence than in those who did after antimuscarinic treatment.

- In patients who did not experience recurrence, urinary NGF/Cr decreased at the end of treatment compared to baseline, and this reduction was maintained at 12 weeks after the end of treatment.

Corresponding author: Kyu-Sung Lee (iD https://orcid.org/0000-0003-0891-2488 Department of Urology, Samsung Medical Center, Sungkyunkwan University School of Medicine, 81 Irwon-ro, Gangnam-gu, Seoul 06351, Korea E-mail: ksleedr@skku.edu / Tel: +82-2-3410-3554 / Fax: +82-2-3410-3027 Submitted: November 19, 2016 / Accepted after revision: March 7, 2017
This is an Open Access article distributed under the terms of the Crecc.
commons.org/licenses/by-nc/4.0/) which permits unrestricted non-commercial use, distribution, and reproduction in any medium, provided the original work is properly cited. 


\section{INTRODUCTION}

Overactive bladder $(\mathrm{OAB})$ is a complex clinical syndrome of urinary urgency, usually accompanied by nocturia and frequency with or without urgency urinary incontinence [1]. Its overall prevalence in males and females older than 40 years is $16.6 \%$ in Europe [2]. Additionally, its prevalence tends to increase with age $[3,4]$. The pathogenesis of OAB may be multifactorial and has yet to be fully clarified [5]. The diagnosis of $\mathrm{OAB}$ and the evaluation of the efficacy of $\mathrm{OAB}$ treatments are usually made based on a careful history and various tools, including voiding diaries and symptom questionnaires. Urodynamic studies can be used in selected cases.

Antimuscarinic treatment for $\mathrm{OAB}$ is usually effective, but the efficacy of treatment varies based on the severity of symptoms and pathophysiology, and 35\% of patients experience recurrence after discontinuing treatment [6]. It is difficult to evaluate the efficacy of antimuscarinic treatment and to determine the adequate duration of treatment. In addition, concerns about the reliability of correlations between symptoms and urodynamic findings have been reported $[7,8]$. Therefore, a new, reliable, and accurate test for evaluating $\mathrm{OAB}$ patients and relevant therapeutic outcomes is needed [9].

Nerve growth factor (NGF) is a neurotrophic factor that induces growth, differentiation, and survival of specific target neurons. NGF is released by smooth muscle and urothelium as a chemical mediator in the bladder [10]. In an animal model, NGF triggered bladder hypersensitivity through the sensitization of afferent nerve pathways [11]. Urinary NGF levels were found to be elevated in $\mathrm{OAB}$ patients in previous studies, and it has been proposed that urinary NGF could be a biomarker of OAB [12-14]. However, studies that investigated urinary NGF as a way of evaluating the efficacy of $\mathrm{OAB}$ treatment have demonstrated inconsistent results $[12,15,16]$. Thus, we investigated changes in urinary NGF levels in subjects with OAB following antimuscarinic treatment in order to predict treatment efficacy and recurrence.

\section{MATERIALS AND METHODS}

\section{Subjects}

This study was approved by the Institutional Review Board of Samsung Medical Center (approval number: 2010-05-106) and was registered at http://clinicaltrials.gov (ClinicalTrials.gov ID: NCT01499069). Written informed consent was obtained from all subjects. We enrolled 189 subjects older than 18 years who visited our outpatient clinic between February 2010 and February 2015 and who had a symptom duration of $\geq 3$ months, with $\geq 8$ micturition events per 24 hours and $\geq 2$ urgency episodes per 24 hours reported in 3-day voiding diaries.

Subjects were excluded if they had stress incontinence, hepatic or renal disease, any condition that is a contraindication for anticholinergic treatment, symptomatic acute or recurrent urinary tract infection, interstitial cystitis, uninvestigated hematuria or hematuria secondary to malignant disease, bladder outlet obstruction, pelvic organ prolapse, bladder cancer or prostate cancer, chronic constipation, treatment with potent CYP3A4 inhibitors, or treatment with any anticholinergic drugs or any drug for $\mathrm{OAB}$ within the 14 days preceding randomization, or if they expected to initiate treatment with such a drug during the study. Subjects also were excluded if they used an unstable dosage of any drug with anticholinergic side effects or expected to start such treatment during the study, if they were pregnant or nursing, or if they had an indwelling catheter or practiced intermittent self-catheterization. We also excluded sexually active females with childbearing potential who were not using reliable contraception, patients with a history of electrostimulation or bladder training within the 14 days before randomization, those who expected to start such treatment during the study, and patients who used any investigational drug within 2 months preceding the start of the study. Patients were also excluded if they had any other condition that, in the opinion of the investigator, made them unsuitable for inclusion.

\section{Study Design}

The study design is summarized in Fig. 1. All subjects with $\mathrm{OAB}$ received antimuscarinic treatment $(4 \mathrm{mg}$ of fesoterodine once daily) and visited our outpatient clinic 4 and 12 weeks (and 24 weeks for patients who completed treatment at 24 weeks) after beginning treatment, as well as 4 and 12 weeks after completing treatment. A 3-day voiding diary, urine samples for evaluating NGF and urinary creatinine (Cr), the Overactive Bladder Questionnaire Short Form (OAB-q SF) symptom bother and health-related quality of life (HRQL) scores, and the overactive bladder symptom score (OABSS) were collected. Urinary levels of NGF were corrected using urinary Cr levels.

Treatment was discontinued at 12 weeks if there was a decrease in micturition events of $>2$ times per 24 hours, a greater than a $50 \%$ decrease in urgency episodes, and if subjects expressed satisfaction on the questionnaire about patients' per- 
OAB subjects

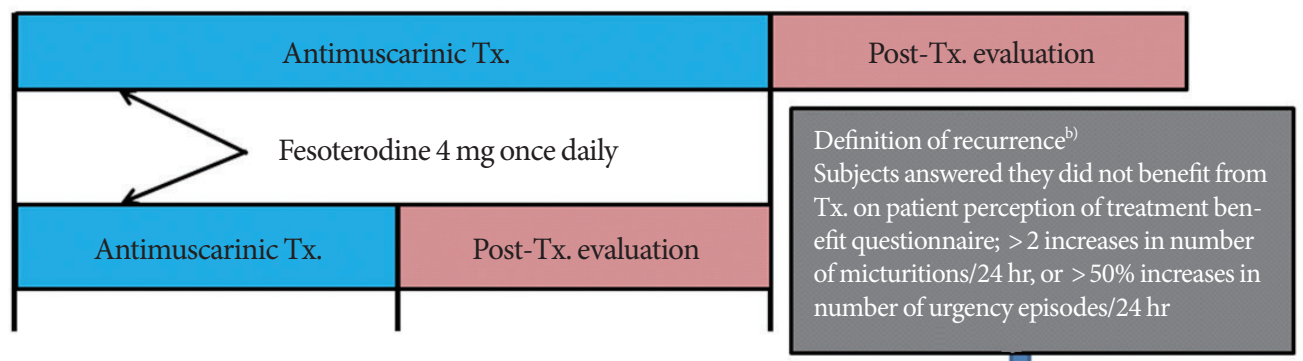

Beginning of Tx.

End of Tx.

End of Tx. ${ }^{a}$

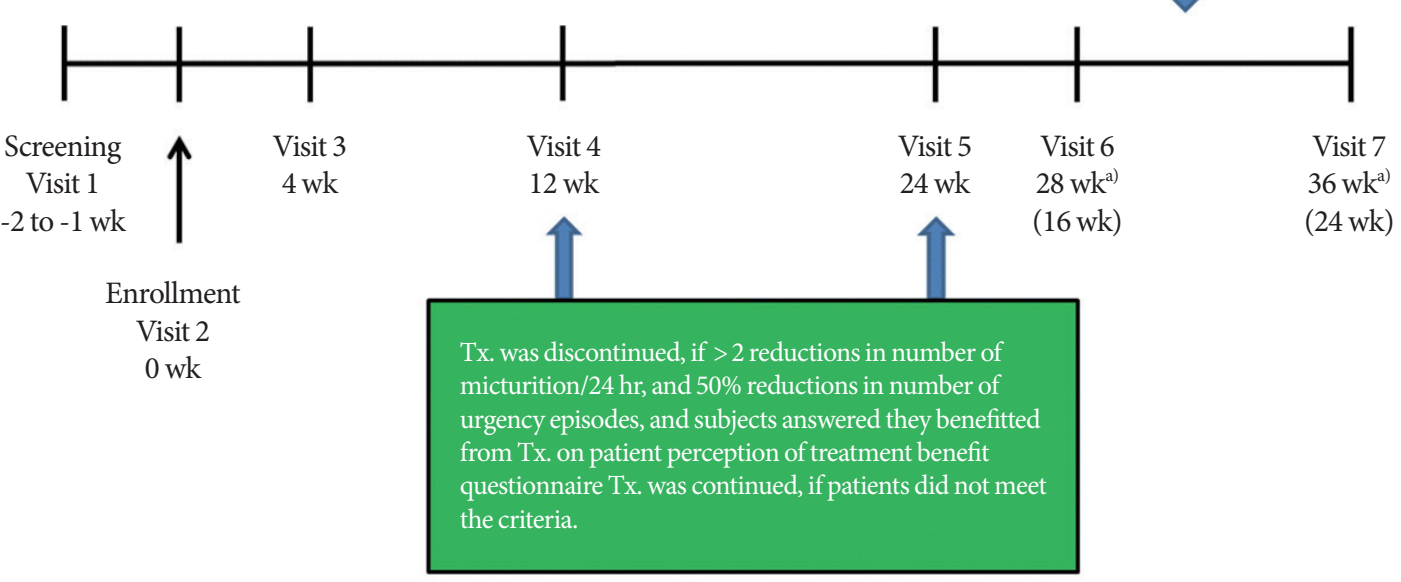

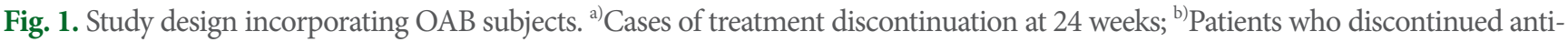
muscarinic treatment at 12 or 24 weeks. OAB, overactive bladder; Tx., treatment.

ceptions of treatment benefits. If subjects did not satisfy these criteria, they received anticholinergics for 12 more weeks and were reevaluated with the same criteria. For subjects who did not meet the criteria at 24 weeks, antimuscarinic treatment was maintained. After completing treatment, subjects who were not satisfied according to the questionnaire on patients' perceptions of treatment benefits and those who had an increase in micturition events of $>2$ times per 24 hours and/or a greater than $50 \%$ increase in urgency episodes were classified as experiencing recurrence.

The baseline characteristics of responders and nonresponders were analyzed according to age, sex, body mass index, $\mathrm{OAB}$ subgroup, $\mathrm{OAB}$ pathogenesis, urinary frequency, urinary urgency, OAB-q SF symptom bother and HRQL, OABSS, and urinary NGF/Cr. Baseline characteristics of patients who did or did not experience recurrence were analyzed in the same manner. Multiple logistic regression analysis was performed in responders and nonresponders and in those who did or did not experience recurrence to identify predictive factors of response to antimuscarinic treatment and recurrence, respectively. Urinary NGF levels after beginning anticholinergic treatment were compared in responders and nonresponders. Urinary NGF levels were also compared after the completion of antimuscarinic treatment in patients who did or did not experience recurrence. A subanalysis was performed according to sex.

\section{Urinary NGF and Cr Measurements}

Voided urine from all subjects was collected at enrollment, at 4 and 12 weeks (and 24 weeks for patients who discontinued treatment at 24 weeks) after the beginning of antimuscarinic treatment, and at 4 and 12 weeks after the completion of treatment. Urine samples were kept on ice and then centrifuged at $3,000 \mathrm{rpm}$ at $4^{\circ} \mathrm{C}$ for 10 minutes. The supernatant was separated into aliquots in $1.5-\mathrm{mL}$ tubes and preserved at $-80^{\circ} \mathrm{C}$. Urinary $\mathrm{Cr}$ was measured in 3-mL urine aliquots.

The urinary NGF concentration was measured using the NGF Emax ImmunoAssay System (Promega Corp., Madison, WI, USA). The wells of 96-well plates were coated with $10 \mu \mathrm{L}$ of 
anti-NGF polyclonal antibody diluted in $10 \mu \mathrm{L}$ of carbonate coating buffer $(\mathrm{pH}, 9.7)$ and incubated at $4^{\circ} \mathrm{C}$ overnight after sealing the wells. Then, the wells were washed 3 times with Tris buffered saline with Tween-20 washing buffer $(20 \mathrm{mM}$ Tris$\mathrm{HCl}, 150 \mathrm{mM} \mathrm{NaCl}$, and $0.05 \%$ Tween 20). Each well was incubated with $200 \mu \mathrm{L}$ of $1 \times$ buffer and sample buffer at room temperature for 1 hour to prevent nonspecific reactions. An NGF standard (prepared from the $1 \mu \mathrm{g} / \mathrm{mL}$ stock solution) and 100 $\mu \mathrm{L}$ of urine were added to the wells. The wells were washed 5 times after incubation for 6 hours, and $100 \mu \mathrm{L}$ of secondary antibody $(2.5 \mu \mathrm{L}$ of anti-NGF monoclonal antibody that was diluted in $10 \mathrm{~mL}$ of $1 \times$ block and sample buffer) was added to each well. The plates were then incubated at $4^{\circ} \mathrm{C}$ overnight. Subsequently, $100 \mu \mathrm{L}$ of anti-rat immunoglobulin G horseradish peroxidase conjugate was added to each well after washing 5 times with TBST washing buffer, and the wells were incubated at room temperature for 2.5 hours. The wells were again washed 5 times with TBST washing buffer. Then, they were incubated with $100 \mu \mathrm{L}$ of tetramethylbenzidine (dissolved in a proprietary buffer) substrate solution at room temperature for 10 minutes. For termination of the reactions, $100 \mu \mathrm{L}$ of $1 \mathrm{~N}$ hydrochloric acid was added. The amount of NGF was determined by the VersaMax ELISA Microplate Reader (Molecular Devices, Inc., Sunnyvale, CA, USA) at $450 \mathrm{~nm}$.

\section{Statistical Analysis}

Data are expressed as mean \pm standard error of the mean. The
Mann-Whitney U-test was used for between-group comparisons. The chi-square test was used for categorical data. Multivariate logistic regression analysis was used to predict response to anticholinergic treatment and recurrence. All variables with a P-value $<0.20$ in the univariate analysis were incorporated into the multivariate analyses. The Wilcoxon signed-rank test with the Bonferroni correction was used for intragroup comparisons. A receiver operating characteristic (ROC) curve was used to analyze the feasibility of NGF as a biomarker. Values of $\mathrm{P}<0.05$ were considered to indicate statistical significance, and all statistical tests were 2 -sided. All statistical analyses were performed using IBM SPSS Statistics ver. 20.0 (IBM Co., Armonk, NY, USA).

\section{RESULTS}

The flow diagram of the progress of all subjects is presented in Fig. 2. Among the 189 enrolled OAB subjects, 92 completed the study. Their mean age was $55.0 \pm 1.5$ years. The percentages of female and male subjects were $78.3 \%$ (72 of 92) and $21.7 \%$ (20 of 92$)$, respectively. The mean number of micturition events per 24 hours was $11.9 \pm 0.3$, and the mean number of urgency episodes per 24 hours was $8.4 \pm 0.5$. The mean OAB-q SF symptom bother score was $20.9 \pm 0.6$, the mean OAB-q SF HRQL score was $42.6 \pm 1.2$, and the mean OABSS was $9.0 \pm 0.2$.

The baseline characteristics of responders $(n=62)$ and nonresponders $(n=30)$ are summarized in Table 1. No variables

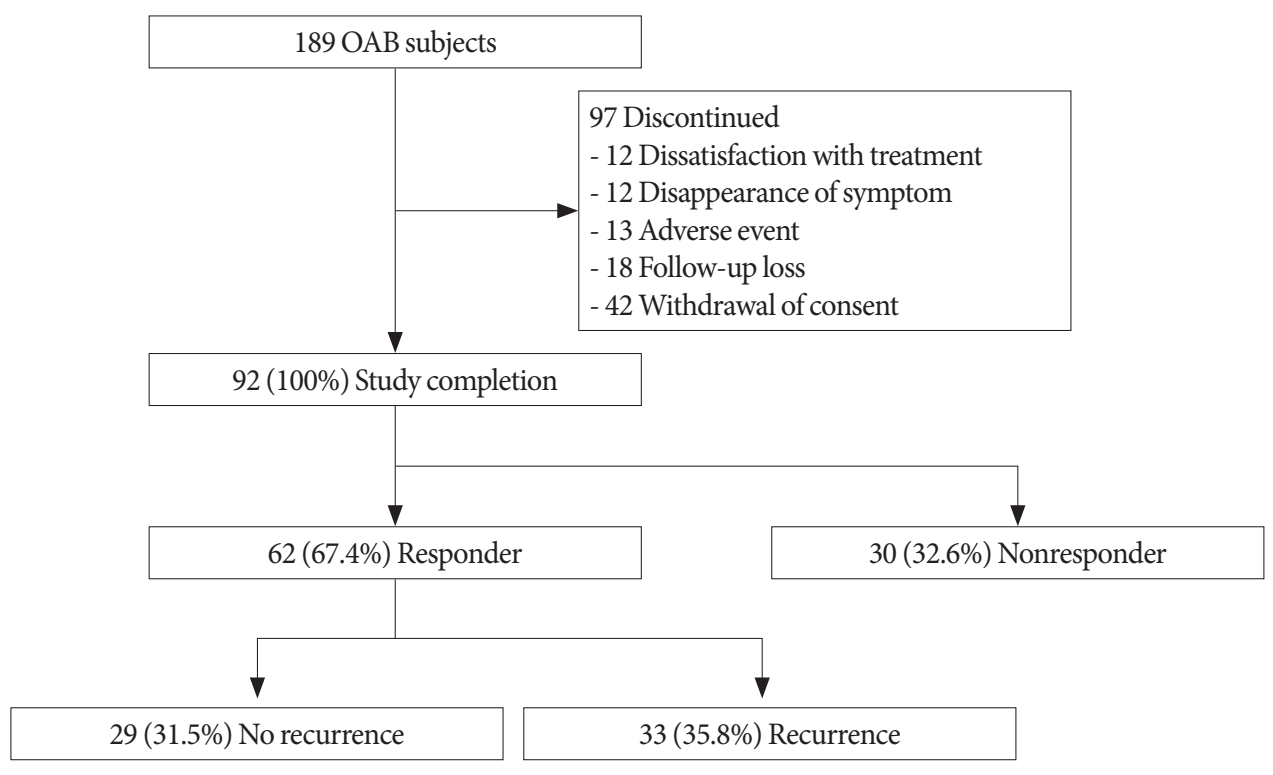

Fig. 2. The flow diagram of the progress of all subjects. OAB, overactive bladder. 


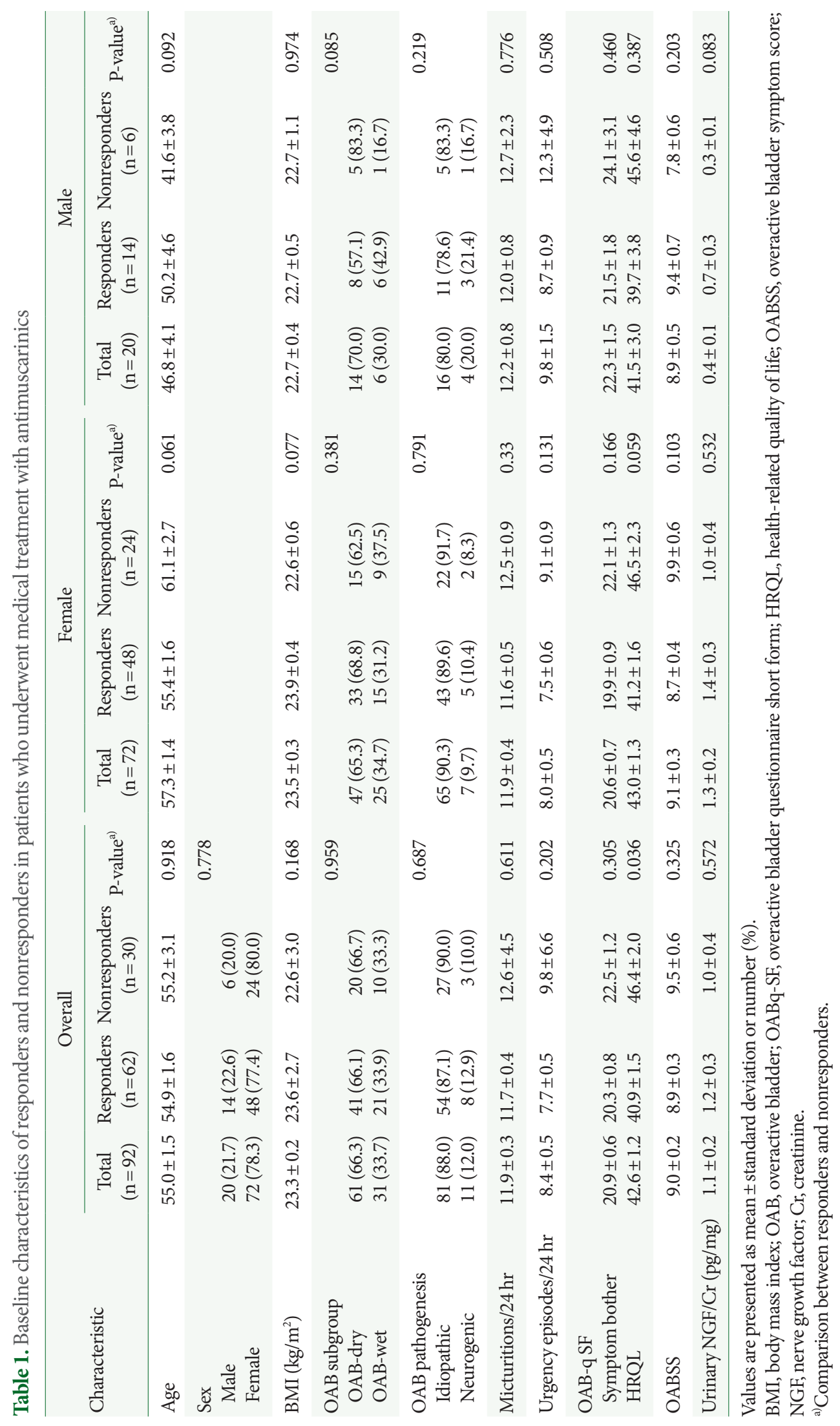




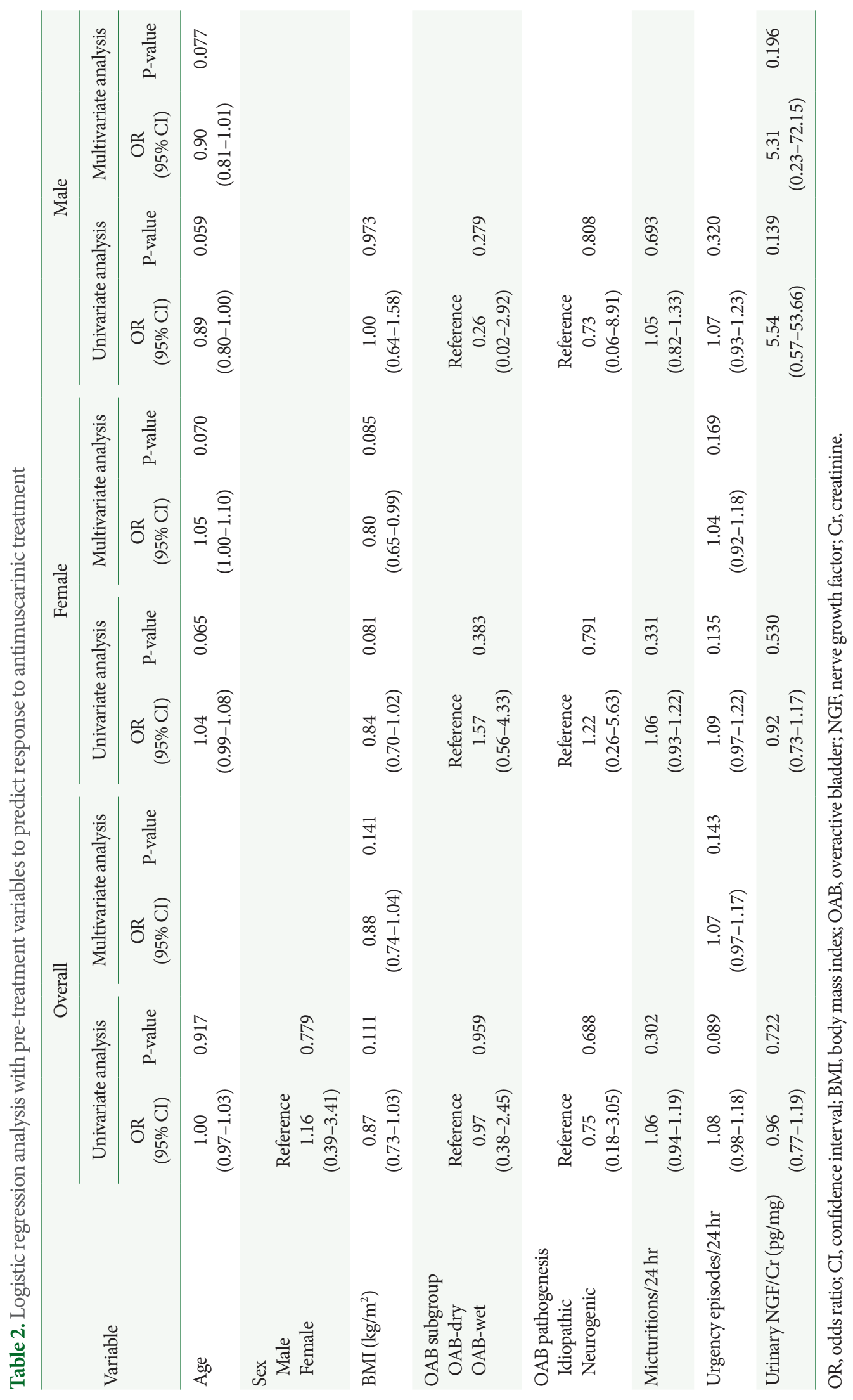




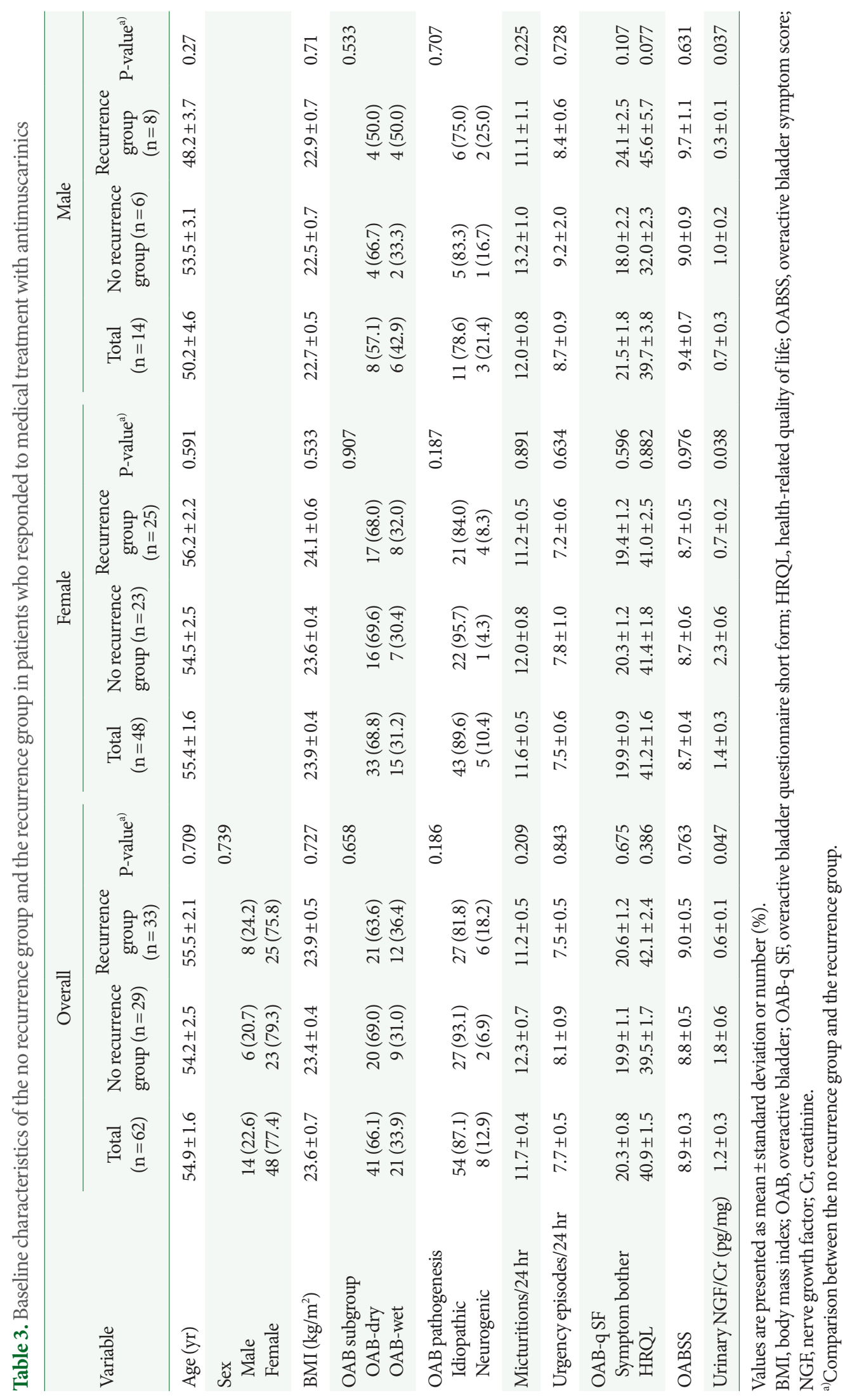




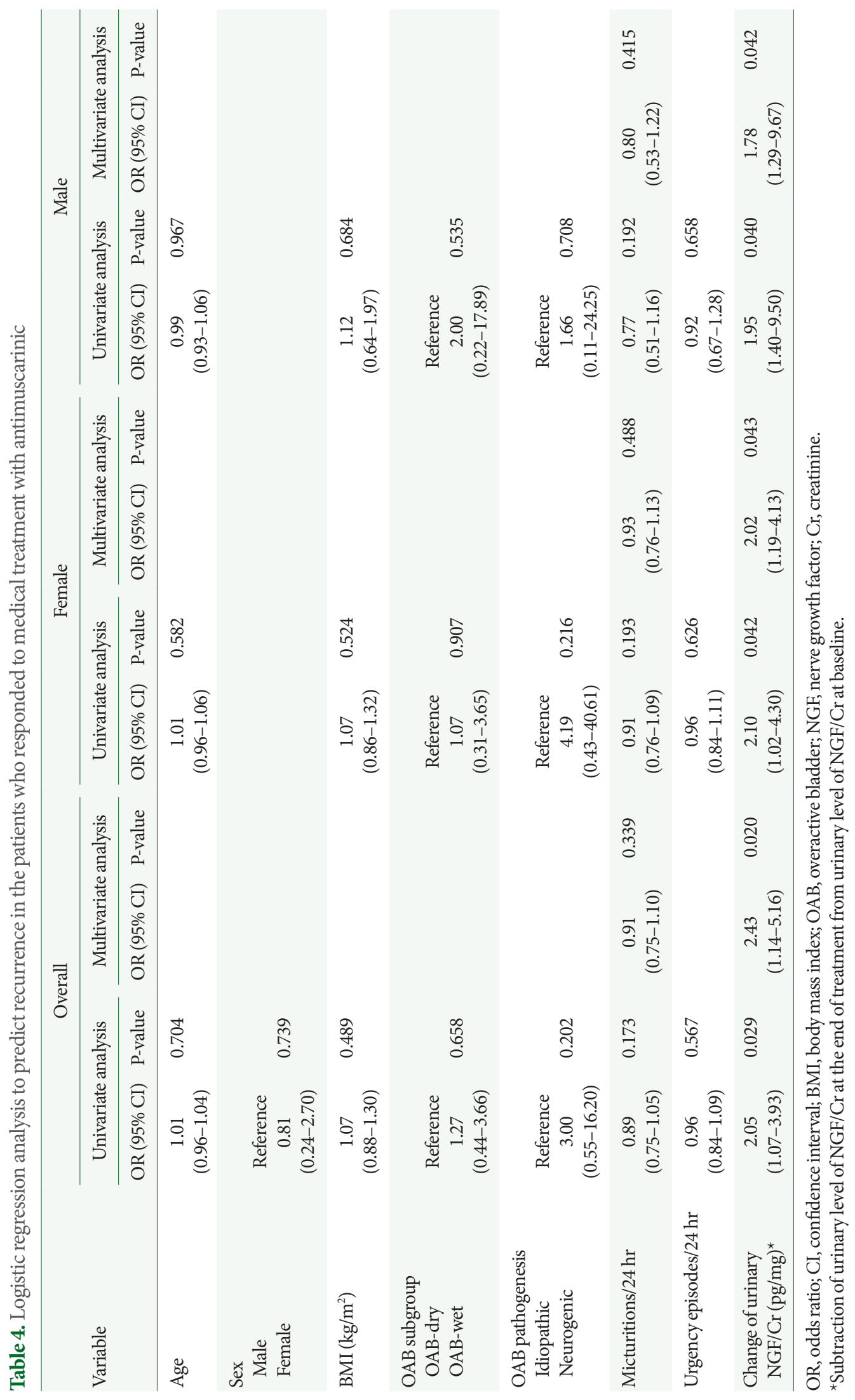


except OAB-q SF HRQL score were significantly different between the 2 groups. No significant difference was found in the subanalysis according to sex. There were no significant predictors of response to antimuscarinic treatment in the logistic regression analysis (Table 2). The baseline characteristics of the no recurrence and recurrence groups in the patients who responded to medical treatment with antimuscarinics are summarized in Table 3. The baseline urinary level of NGF/Cr was
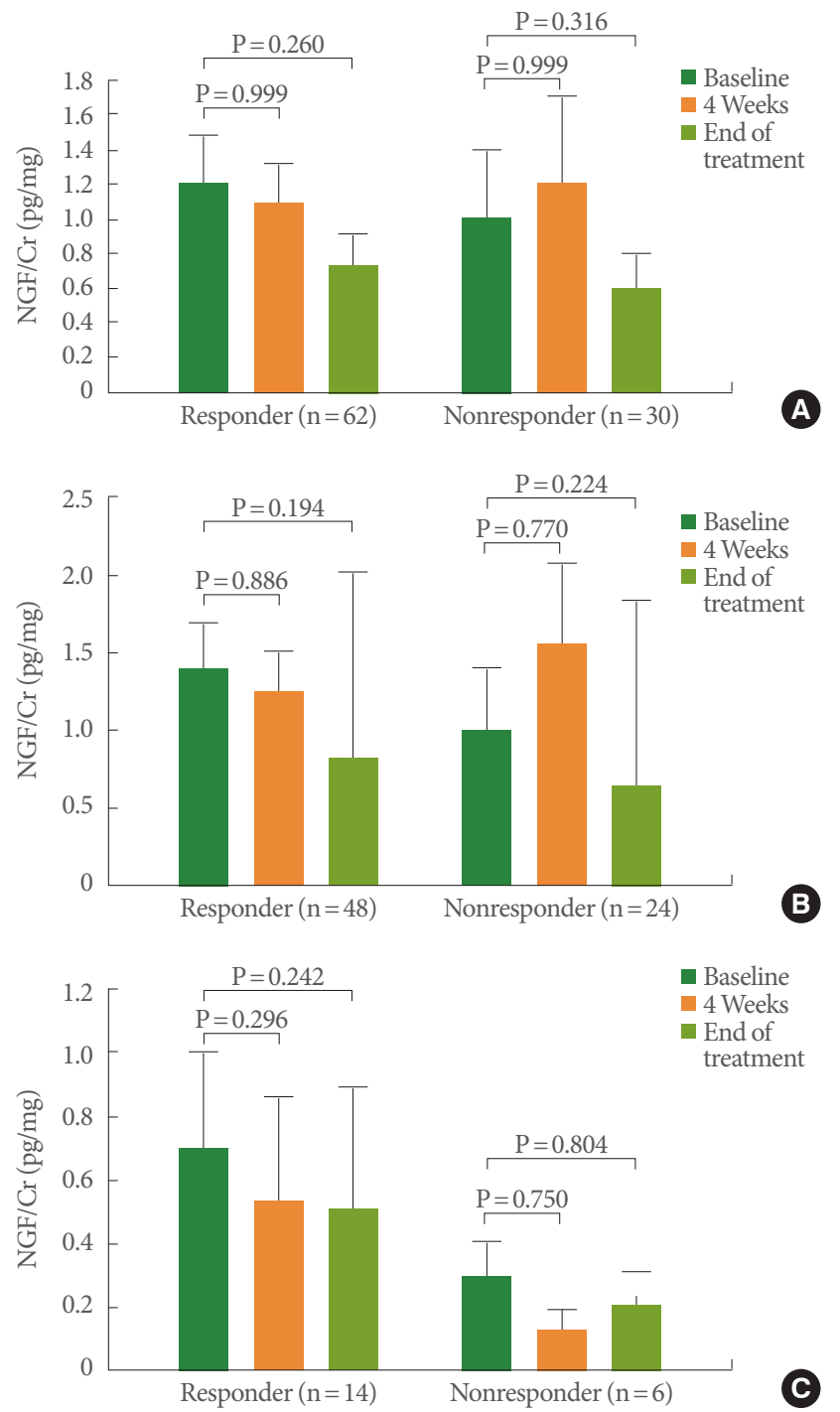

Fig. 3. Urinary NGF levels after the beginning of antimuscarinic treatment in responders and nonresponders among patients with OAB. Overall (A), female (B), and male (C) subjects. Columns are mean \pm standard error of the mean. The time of the end of treatment was 12 or 24 weeks for responders and 24 weeks for nonresponders. NGF, nerve growth factor; Cr, creatinine; $\mathrm{OAB}$, overactive bladder. higher in those who did not experience recurrence than in those who did $(\mathrm{P}=0.047)$. This finding was also noted in the subanalysis of female and male subjects. In the logistic regression analysis, change in the urinary level of NGF/Cr (evaluated by subtracting the urinary level of NGF/Cr at baseline from the urinary level of NGF/Cr at the completion of treatment) was a significant predictor of recurrence regardless of sex (Table 4).

Among responders $(n=62)$, the urinary level of NGF/Cr
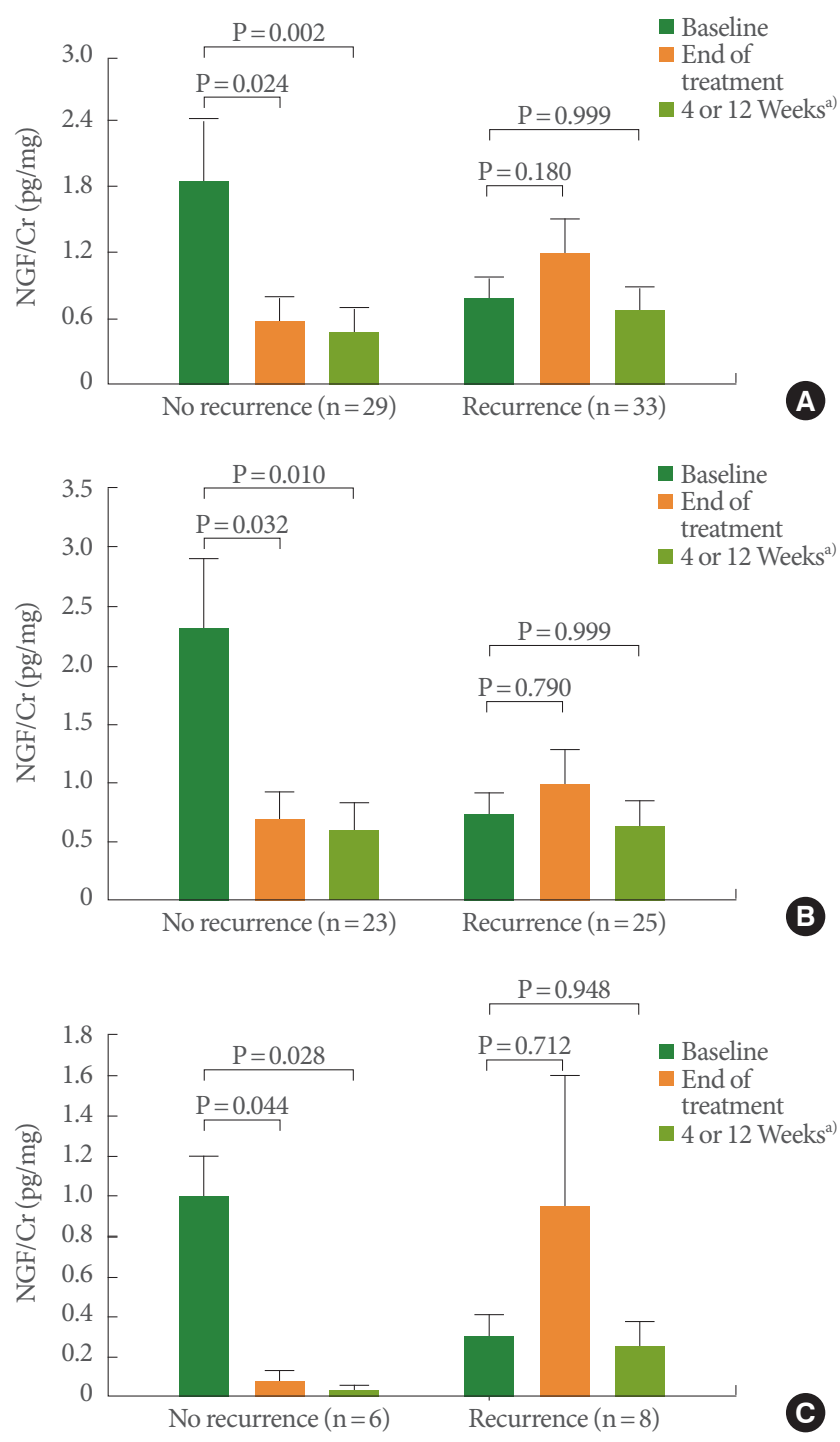

Fig. 4. Urinary NGF levels at baseline and after the end of antimuscarinic treatment in the $\mathrm{OAB}$ subjects who did or did not experience recurrence. Overall (A), female (B), and male (C) subjects. Columns are mean \pm standard error of the mean. ${ }^{\text {a) }} 12$ Weeks after end of treatment in the no recurrence group and at the time of recurrence after end of treatment in the recurrence group. NGF, nerve growth factor; $\mathrm{OAB}$, overactive bladder; $\mathrm{Cr}$, creatinine. 
tended to decrease, but there was no significant change after beginning antimuscarinic treatment (Fig. 3). Among those who did not experience recurrence $(n=29)$, the urinary level of $\mathrm{NGF} / \mathrm{Cr}$ after treatment decreased compared to baseline $(\mathrm{P}=0.024)$ and remained significantly decreased 12 weeks after the end of treatment $(\mathrm{P}=0.002)$. The recurrence group $(\mathrm{n}=33)$ did not show any significant change in the urinary level of $\mathrm{NGF/Cr}$ (Fig. 4). These results were also found in the subanalysis according to sex.

In the ROC curve analysis of urinary NGF/Cr among responders to antimuscarinic treatment, change in the urinary level of NGF/Cr (evaluated by subtracting the urinary level of $\mathrm{NGF} / \mathrm{Cr}$ at baseline from the urinary level of NGF/Cr at the completion of treatment) discriminated the no recurrence group from the recurrence group in the subjects overall, in female subjects, and in male subjects (area under the curve $[\mathrm{AUC}]=0.65 ; 95 \%$ confidence interval $[\mathrm{CI}], 0.52-0.80 ; \mathrm{P}=$ 0.043; $\mathrm{AUC}=0.66 ; 95 \% \mathrm{CI}, 0.55-0.80 ; \mathrm{P}=0.037 ; \mathrm{AUC}=0.63$; $95 \% \mathrm{CI}, 0.52-0.77 ; \mathrm{P}=0.045$, respectively).

\section{DISCUSSION}

In this study, we investigated the potential of urinary NGF as a biomarker for prediction of the efficacy of antimuscarinic treatment and recurrence. In previous studies, urinary NGF/Cr levels significantly decreased after antimuscarinic treatment [12], and were significantly reduced in responders in other studies $[13,15]$. However, we observed different results. The present study demonstrated that urinary NGF/Cr levels tended to decrease in responders, including both recurrent and nonrecurrent patients, but this change was not statistically significant. Patients who did not experience recurrence showed a significant decrease in urinary NGF/Cr levels after treatment. Urinary NGF/Cr appeared to decrease only in those who did not experience recurrence. This finding is the main strength of our study, suggesting that medication cessation should be considered only in those who maintain lowered urinary NGF/Cr levels compared to baseline by the end of treatment. Interestingly, in the recurrence group, the urinary NGF/Cr level showed a tendency to increase after treatment, despite the response to antimuscarinics. This result implies that an unknown mechanism of anticholinergics may have been present in the no recurrence group. The mechanism through which the urinary level of NGF increases in OAB patients is not yet established. Stretching of the bladder smooth muscle cells increased the ex- pression of messenger RNA for NGF and stimulated the secretion of NGF in vitro [17]. A decreased urinary level of NGF after antimuscarinic treatment might be related to the suppression of bladder smooth muscle as a result of OAB symptom improvement [15]. Further studies to confirm these results, as well as research into the precise mechanism, are required.

The finding that a significant decrease in urinary NGF/Cr is predictive that no recurrence will take place after treatment could help physicians to make the decision to stop antimuscarinic treatment. In the present study, a change in the urinary levels of NGF/Cr was revealed as a potential test for identifying patients who will not experience recurrence (AUC $=0.65 ; 95 \%$ CI, $0.52-0.80 ; \mathrm{P}=0.043$ ). Although the discrimination power is not particularly high, this finding is of value considering that there is currently no definite way to decide when to stop antimuscarinic treatment in responders.

Increased urinary NGF levels have been reported in various lower urinary tract dysfunctions. Urinary levels of NGF/Cr were elevated in patients with interstitial cystitis/bladder pain syndrome [18]. Elevated urinary NGF levels were not observed in patients with urodynamic stress urinary incontinence [19], but increased urinary NGF levels were reported in a rat model of stress urinary incontinence [20]. Bladder outlet obstruction stimulated an increase in urinary NGF [21]. Elevated urinary NGF levels have also been found in other conditions, including stones, urinary tract infections, and bladder tumors [18,22]. Additionally, a relatively wide variation in urinary NGF was found in the present study. The fact that duplicate urinary samples were not analyzed when measuring urinary NGF and Cr to minimize the variation is a limitation of this study. Although NGF may have some limitations as a biomarker of OAB, we believe that urinary NGF levels may be useful for evaluating treatment outcomes in clinically diagnosed OAB patients.

Along with NGF, other neurotrophic factors, such as brain-derived neurotrophic factor (BDNF), and glial cell line-derived neurotrophic factor (GDNF) have also been investigated as biomarkers for OAB. BDNF is expressed in the urothelium and exerts an influence through TrkB receptors [23]. The nonpeptidergic subpopulation is regulated by GDNF, which binds to GDNF family receptor $\alpha 1[24,25]$. Urinary BDNF levels were significantly higher in $\mathrm{OAB}$ patients than in healthy controls and were normalized after the treatment of $\mathrm{OAB}$, while urinary GDNF levels did not significantly differ between those 2 groups $[12,23,26]$. These data suggest that BDNF is also an important element in the pathogenesis of OAB. Further studies of urinary neurotrophins, 
including NGF and BDNF, are required for understanding the pathogenesis and improving the treatment of $\mathrm{OAB}$.

Of note, the Promega (Madison, WI, USA) discontinued production of the NGF Emax ImmunoAssay System on March 15,2014 , during the study period. However, the measurement of urinary NGF was not interrupted in this study, as our stock was sufficient. Thus, measurements of NGF were performed consistently with the same kit.

Recently, questions about the suitability of the Promega assay for the detection of NGF in urine have been raised [27]. Researchers reported cross-reactions of the assay with urinary compounds, which could be another limitation of the present study. Although this is an important concern about the validity of the Promega assay that should be clarified, we believe that our serial data of urinary NGF/Cr in each patient nonetheless show the potential of urinary NGF as a biomarker of treatment outcomes. Future studies to confirm our findings using other assays are needed.

We report the novel outcome of urinary NGF being useful as a potential biomarker for predicting the outcome of antimuscarinic treatment in $\mathrm{OAB}$ patients in this study. To the best of our knowledge, the finding of a decrease in urinary NGF/Cr only among patients who did not experience recurrence has not been reported in previous studies. This may provide useful information regarding the decision of when to stop antimuscarinic treatment in responders.

In conclusion, urinary NGF/Cr levels tended to decrease after antimuscarinic treatment, but this was not significant. Those who did not experience recurrence had higher baseline urinary $\mathrm{NGF} / \mathrm{Cr}$ levels than the recurrence group. In those who did not experience recurrence, urinary NGF/Cr decreased at the completion of treatment compared to baseline, and this was maintained for 12 weeks after completion of treatment.

These data suggest that urinary NGF can serve as a potential biomarker for predicting treatment outcomes in $\mathrm{OAB}$ patients. This may provide useful information regarding the decision of when to stop antimuscarinic treatment in responders.

\section{REFERENCES}

1. Haylen BT, de Ridder D, Freeman RM, Swift SE, Berghmans B, Lee J, et al. An International Urogynecological Association (IUGA)/International Continence Society (ICS) joint report on the terminology for female pelvic floor dysfunction. Int Urogynecol J 2010;21:526.
2. Stewart WF, Van Rooyen JB, Cundiff GW, Abrams P, Herzog AR, Corey R, et al. Prevalence and burden of overactive bladder in the United States. World J Urol 2003;20:327-36.

3. Lee YS, Lee KS, Jung JH, Han DH, Oh SJ, Seo JT, et al. Prevalence of overactive bladder, urinary incontinence, and lower urinary tract symptoms: results of Korean EPIC study. World J Urol 2011; 29:185-90.

4. Sever N, Oskay U. An investigation of lower urinary tract symptoms in women aged 40 and over. Low Urin Tract Symptoms 2017; 9:21-6.

5. Silva-Ramos M, Silva I, Oliveira O, Ferreira S, Reis MJ, Oliveira JC, et al. Urinary ATP may be a dynamic biomarker of detrusor overactivity in women with overactive bladder syndrome. PLoS One 2013;8:e64696.

6. Choo MS, Song C, Kim JH, Choi JB, Lee JY, Chung BS, et al. Changes in overactive bladder symptoms after discontinuation of successful 3-month treatment with an antimuscarinic agent: a prospective trial. J Urol 2005;174:201-4.

7. Hashim H, Abrams P. Is the bladder a reliable witness for predicting detrusor overactivity? J Urol 2006;175:191-4.

8. Digesu GA, Khullar V, Panayi D, Calandrini M, Gannon M, Nicolini U. Should we explain lower urinary tract symptoms to patients? Neurourol Urodyn 2008;27:368-71.

9. Fry CH, Sahai A, Vahabi B, Kanai AJ, Birder LA. What is the role for biomarkers for lower urinary tract disorders? ICI-RS 2013. Neurourol Urodyn 2014;33:602-5.

10. Steers WD, Tuttle JB. Mechanisms of Disease: the role of nerve growth factor in the pathophysiology of bladder disorders. Nat Clin Pract Urol 2006;3:101-10.

11. Chuang YC, Fraser MO, Yu Y, Chancellor MB, de Groat WC, Yoshimura $\mathrm{N}$. The role of bladder afferent pathways in bladder hyperactivity induced by the intravesical administration of nerve growth factor. J Urol 2001;165:975-9.

12. Antunes-Lopes T, Pinto R, Barros SC, Botelho F, Silva CM, Cruz $\mathrm{CD}$, et al. Urinary neurotrophic factors in healthy individuals and patients with overactive bladder. J Urol 2013;189:359-65.

13. Kuo HC, Liu HT, Chancellor MB. Can urinary nerve growth factor be a biomarker for overactive bladder? Rev Urol 2010;12:e69-77.

14. Liu HT, Chancellor MB, Kuo HC. Urinary nerve growth factor levels are elevated in patients with detrusor overactivity and decreased in responders to detrusor botulinum toxin-A injection. Eur Urol 2009;56:700-6.

15. Kim SR, Moon YJ, Kim SK, Bai SW. NGF and HB-EGF: potential biomarkers that reflect the effects of fesoterodine in patients with overactive bladder syndrome. Yonsei Med J 2015;56:204-11. 
16. Vijaya G, Cartwright R, Bhide A, Derpapas A, Fernando R, Khullar $\mathrm{V}$. Reliability and validity of urinary nerve growth factor measurement in women with lower urinary tract symptoms. Neurourol Urodyn 2016;35:944-8.

17. Persson K, Sando JJ, Tuttle JB, Steers WD. Protein kinase C in cyclic stretch-induced nerve growth factor production by urinary tract smooth muscle cells. Am J Physiol 1995;269(4 Pt 1):C101824.

18. Liu HT, Tyagi P, Chancellor MB, Kuo HC. Urinary nerve growth factor but not prostaglandin E2 increases in patients with interstitial cystitis/bladder pain syndrome and detrusor overactivity. BJU Int 2010;106:1681-5.

19. Liu HT, Chancellor MB, Kuo HC. Urinary nerve growth factor level could be a biomarker in the differential diagnosis of mixed urinary incontinence in women. BJU Int 2008;102:1440-4.

20. Ko IG, Kim SE, Kim BK, Shin MS, Kim CJ, Yim SJ, et al. Swimming: effects on stress urinary incontinence and the expression of nerve growth factor in rats following transabdominal urethrolysis. Int Neurourol J 2011;15:74-81.
21. Steers WD, Kolbeck S, Creedon D, Tuttle JB. Nerve growth factor in the urinary bladder of the adult regulates neuronal form and function. J Clin Invest 1991;88:1709-15.

22. Liu HT, Chen CY, Kuo HC. Urinary nerve growth factor levels in overactive bladder syndrome and lower urinary tract disorders. J Formos Med Assoc 2010;109:862-78.

23. Bhide AA, Cartwright R, Khullar V, Digesu GA. Biomarkers in overactive bladder. Int Urogynecol J 2013;24:1065-72.

24. Ochodnický P, Cruz CD, Yoshimura N, Michel MC. Nerve growth factor in bladder dysfunction: contributing factor, biomarker, and therapeutic target. Neurourol Urodyn 2011;30:1227-41.

25. Sariola H, Saarma M. Novel functions and signalling pathways for GDNF. J Cell Sci 2003;116(Pt 19):3855-62.

26. Antunes-Lopes T, Carvalho-Barros S, Cruz CD, Cruz F, MartinsSilva C. Biomarkers in overactive bladder: a new objective and noninvasive tool? Adv Urol 2011;2011:382431.

27. Gamper M, Moser R, Viereck V. Have we been led astray by the NGF biomarker data? Neurourol Urodyn 2017;36:203-4. 